\title{
Molecular Electronics Device Modeling for System Design
}

\author{
Ci Lei*, Dinesh Pamunuwa*, Steven Bailey ${ }^{\dagger}$ and Colin Lambert ${ }^{\dagger}$ \\ ${ }^{*}$ Center for Microsystems Engineering, Department of Engineering \\ Lancaster University, Lancaster, UK \\ †Condensed Matter Theory Group, Department of Physics \\ Lancaster University, Lancaster, UK
}

\begin{abstract}
We have used the Breit-Wigner resonance formula to model a class of molecular electronics devices with the aim to establish an abstract model for a molecular electronic device that can be used in a general cross bar architecture of future nanoelectronic systems. We show that the molecular I-V curves can be characterized by a very small number of variables including the couplings between the contact and leads.
\end{abstract}

\section{INTRODUCTION}

Molecular conductors are of great interest from both a theoretical and an applied electronics point of view. The present objective in this field is to develop and control electronic molecules that can serve as the active elements in future nano-electronic circuits. Recently researchers in this area have made significant progress, demonstrating devices with negative differential resistance [1], electrically configurable switches [2] and single molecule transistors [3]-[5]. Although developing a reliable molecular-electronics device still has many key problems such as device stability, reproducibility and the effective control of single-molecule transport, many research groups have begun to examine possible system architectures which can use these devices to their best advantage. Fully functional nano-circuits such as molecular memories [2] and logic gates [6]-[8] have already been demonstrated in the lab, but a system architecture that allows efficient communication between submodules and with external circuits is required for a complete nanoelectronics system. One of the most promising architectures is a crossbar scheme [9]-[13] - an abstract representation of which is shown in Fig.3 - due to its advantages in size, fault tolerance and easy fabrication. While there have been significant efforts aimed at investigating the nano-scale crossbar architecture at the device and architectural levels, little consideration has been given to the intermediate levels of abstraction. A hierarchy of device and interconnect models are needed, as the molecular electronics devices are combined in large structures and performance prediction of each individual device within the system becomes essential for any sort of system design. Current modeling techniques for molecular conduction employ either semi-empirical or ab initio methods. The ab initio method is computationally quite expensive even for small molecules and is not suitable for design or analyses of large systems. As part of current research work aiming to develop behavioural models for molecular electronics devices, this paper reports an abstract model which

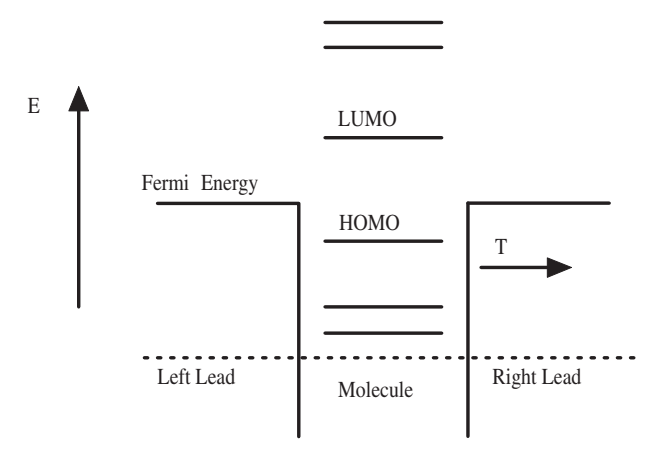

Fig. 1. A schematic energy diagram shows energy level structure of the device at zero bias voltage(equilibrium case). The leads couple to the energy levels of the molecule and electrons are incident from the left lead at its Fermi energy. They scatter thought the molecule and have a transmission probability $T$, to enter the right lead.

can be used in circuit simulation of nano circuits constructed using the crossbar architecture.

\section{Methodology}

In molecular electronics, a molecular transport junction containing one or more molecules extending between two electrodes has been recognized as a very important structure in the construction of molecular devices. In this context, understanding of the electron transport of a single molecule is extremely important. The electron transport properties of a molecular system can be studied by viewing it as a oneelectron elastic scattering problem [14]. In this scattering model, the electrodes are treated as electron reservoirs and the molecule is treated as a scattering impurity between the two electrodes. An incoming electron from the source lead with an energy $E$, has a transmission probability $T(E)$ describing the ease with which electrons can scatter through the molecule into the drain lead. A schematic energy diagram of the model system is shown in Fig. 1.

The transmitted current $I$ through the molecule is proportional to the transmission probability for a range of energies around the fermi energy of the source lead, and it is computed using Landauer's formula [15],

$$
I(V)=\frac{2 e}{h} \int_{-\infty}^{\infty} d E T(E)\left(\frac{1}{\exp \left[\left(E-\mu_{s}\right) / k T\right]+1}\right.
$$




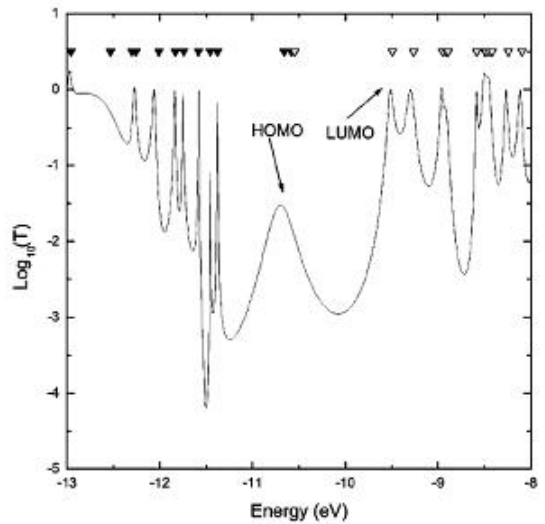

Fig. 2. Transmission function of the T3 molecule calculated by the ESQC technique. HOMO and LUMO refer to the gap of T3 adsorbed on the two electrodes. The energy scale reference is arbitrary [17].

$$
\left.-\frac{1}{\exp \left[\left(E-\mu_{d}\right) / k T\right]+1}\right) .
$$

The two electro-chemical potentials $\mu_{s}$ and $\mu_{d}$ refer to the source and drain respectively. For the symmetric molecules, they are defined to be,

$$
\begin{gathered}
\mu_{s}=E_{f}+e V / 2, \\
\mu_{d}=E_{f}-e V / 2,
\end{gathered}
$$

where $V$ is the bias voltage applied between the source and drain, and $E_{f}$ is the Fermi energy, which is set to $E_{f}=0$ in all figures.

One way to calculate the transmission probability $T(E)$ used in the Landauer formula is to solve the Schrodinger equation directly for the scattered wavefunction of the electron using the non-equilibrium Green's function method [16]. This method will approximate the infinite electrode-moleculeelectrode system by a finite structure confined in a device region. The plane wave of the electron incident from the source lead will give rise to a reflected wave back into the various modes of the source lead. When the energy of molecular orbitals is in resonance with tunneling electrons, some of the wave will be transmitted through the molecule and into the modes of the drain lead. The scattered wavefunction enables the determination of $T(E)$, ie., how much was transmitted. In Fig.2, we show an example of the transmission function calculated using the elastic scattering quantum chemistry technique [17].

The electronic properties of the molecules are significantly affected by the nature of the electrode-molecule coupling. If we consider the case where particular energy levels of the molecule do not overlap with each other (ie., weak moleculeelectrode bonding), it is well known that the transmission probability can be approximated by the Breit-Wigner formula [18],

$$
T(E)=\frac{4 \Gamma_{1} \Gamma_{2}}{\left(E-\varepsilon_{0}\right)^{2}+\left(\Gamma_{1}+\Gamma_{2}\right)^{2}}
$$

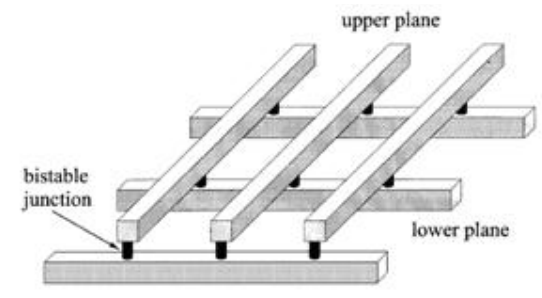

Fig. 3. The crossbar paradigm consists of perpendicular sets of parallel wires with bistable junctions at each wire crossing [9].

where the variables $\Gamma_{1}$ and $\Gamma_{2}$ represent the broadenings of molecular levels by hybridization with the contacts and the molecular level $\varepsilon_{0}$ is related to the intrinsic chemistry of the molecule. This Breit-Wigner formula provides a convenient framework for device modeling of nanoscale molecular electronics at the design level abstraction.

Starting with isolated devices, we can employ it in a two-dimensional crossbar circuit which is a pathway towards increasingly complex circuits. The crossbar circuit architecture is chosen due to its several advantages. Firstly, a crossbar with $2^{N}$ nanowires may be individually addressed by only $2 N$ communication wires, hence the communication between the nano-circuit and external circuit will be efficient. Secondly, the crossbar architecture enables the construction of a largescale generic circuit that can be electronically configured for memory, logic applications, or signal routing applications. The number of wires can be increased arbitrarily as the wire dimensions scale continuously down to molecular sizes. Thirdly, the stochastic process behind molecular self-assembly will inevitably give rise to defects in the manufactured structures, and the reconfigurable architecture of the crossbar is defect tolerant. Finally, the simple physical structure of the crossbar involves only two sets of straight, aligned wires. This enables the crossbars to be fabricated using a vide variety of techniques and makes nanoscale fabrication feasible and potentially inexpensive. Fig. 3 shows an abstract representation of such crossbar, consisting of two parallel planes of nanoscale wire arrays crossing perpendicularly, with each pair of planes separated by a thin layer of a chemical species with particular electrochemical properties.

\section{RESULTS AND ANALYSIS}

To validate our proposal we simulated various crossbar circuits using the proposed device model to investigate the output voltage $V_{\text {out }}$. For the device, we consider a system which consists of an extended molecule contacted between semi-infinite leads. These leads are coupled to the ends of the molecule. In Fig.4, we plot the total transmission as a function of molecular energy for various resonant energies and various resonant widths. Figs. 5 and 6 show the calculated current through the molecule using Eq.(1), with the I-V curves being characterized by different $\Gamma_{1}$ and $\Gamma_{2}$. From the I$\mathrm{V}$ characteristics, it is evident that the device has a valid operating range for switch-like behavior. Within this range, the 


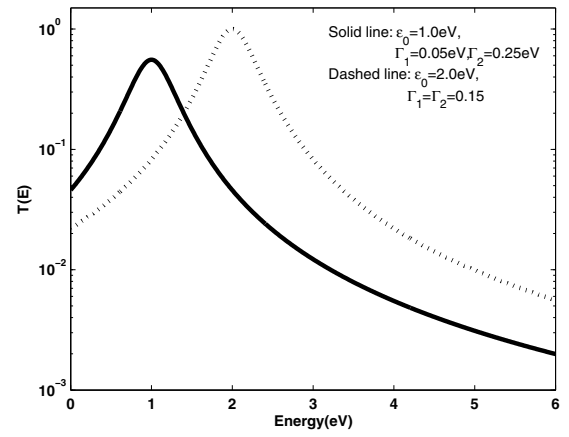

Fig. 4. Total transmission as a function of molecular energy for various molecular energy levels.

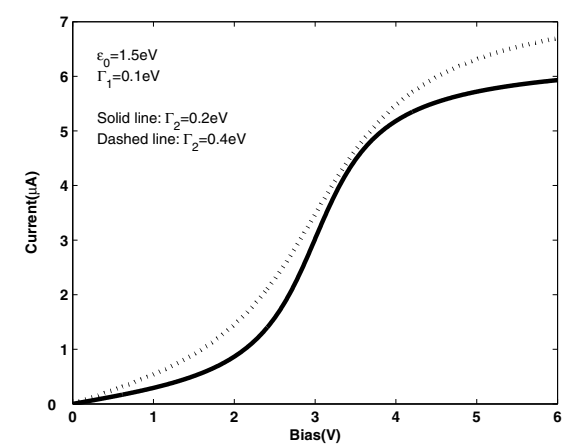

Fig. 5. Calculated I-V characteristics for different resonant width using Eq.1.

device will stay in one of two states: high impedance ('open') or low impedance ('close'). The device in one state will switch to the other state if the voltage drop across the device exceeds the values corresponding to the close/open states. Excessive positive or negative voltage drops across the device need to be avoided.

The logic circuit we consider is shown in Fig.7a which is the $A+B C$ boolean function. Fig.7b shows the molecular electronics crossbar implementation, with the horizontal and vertical lines representing nanowires. The horizontal wires are located in one plane and vertical wires in another. This is an architecture designed for crosspoint addressing, with the input vector on the row wires and the output presented vertically on the column wires. The inputs are labeled alphabetically. For this logic gate, a low input is held at ground, and a high input is held at $+5 \mathrm{~V}$. The schematic shows explicit pull-up and pulldown resistors, labeled as $R_{u p}$ and $R_{\text {down }}$, respectively. For all simulations, $R_{u p}$ and $R_{\text {down }}$ are both set to $50 k \Omega$. Selected junctions in this circuit are configured to supply the pull-up and pull-down resistors required by different logic functions. Each junction consists of a extended molecule sandwiched between metal electrodes. The I-V characteristics of this device is modeled using the formula described in Section II with the variables $\Gamma_{1}=0.1 \mathrm{eV}, \Gamma_{2}=0.2 \mathrm{eV}$ and $\varepsilon_{0}=1.5 \mathrm{eV}$. At the current stage, all simulations are performed at a low temperature. As the temperature goes high, the outputs become

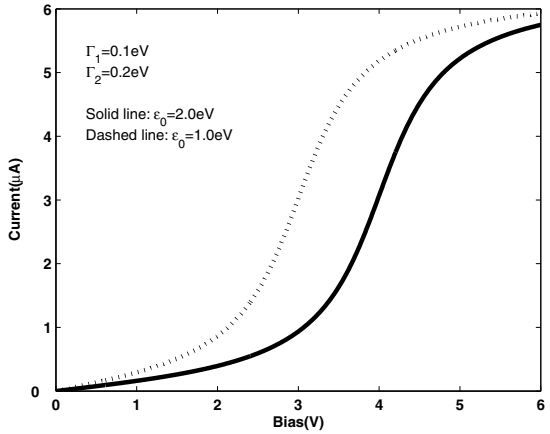

Fig. 6. Calculated I-V characteristics for different molecular energy levels using Eq.1.
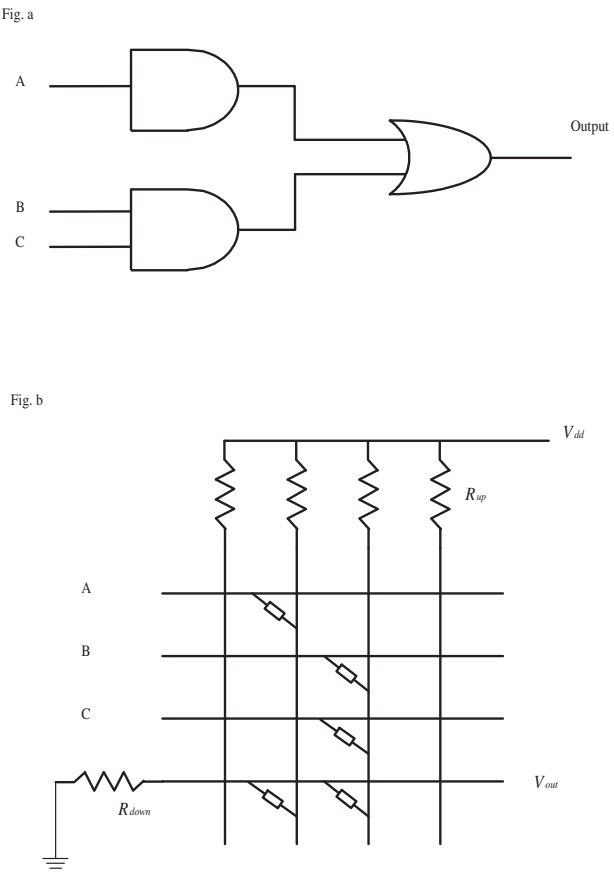

Fig. 7. An implementation of an AND/OR gate in the crossbar circuit

less significant. A truth table derived for a two-input AND is shown in Fig.(8). A high response $V_{\text {out }}=2.76 \mathrm{~V}$ is recorded when both inputs are high. A low response $V_{\text {out }}=0.55 \mathrm{~V}$ is recorded when both inputs are low. In Fig.(9), we present the truth table for a two-input OR gate. In this case, if any input combination is held high, then the output is a high response with $V_{\text {out }} \geq 1.56 \mathrm{~V}$. A low response $V_{\text {out }}=0.7 \mathrm{~V}$ is recorded when both inputs are low. Finally, In Fig.(10), the truth table for the three-input function $A+B C$ is presented.

\section{CONCLUding Remarks}

The simulation of the circuits with molecular electronics device requires the development of a molecular device model, which will not only accurately represent the input-output characteristics of the molecular device, but will also be suitable for implementation in an existing circuit simulator, like SPICE [19]-[21]. 


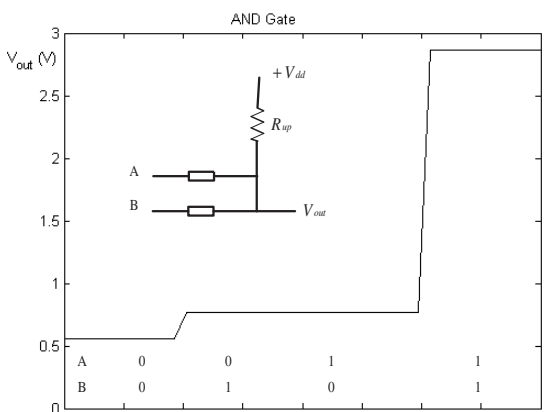

Fig. 8. A derived truth table for a two-input AND gate

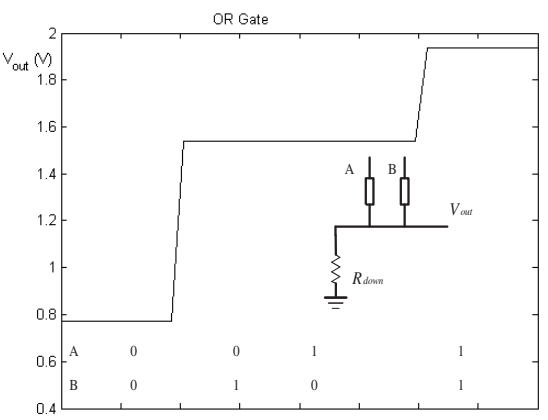

Fig. 9. A derived truth table for a two-input OR gate

In this paper, we have proposed a simple model for general molecule electronics device to be used in a general cross bar architecture of future nanoelectronic systems, when the molecule is weakly coupled to the electrodes. In our model, the Breit-Wigner formula for the resonant conductance is used to characterize the shape of current-voltage curves which are determined by the combined effect of the electronic structure of the molecule. There are some limitations of our model that need to be addressed. The presented model is restricted to the weak coupling limit. It is not viable when the width of the molecular orbitals is comparable with energy difference between them, which may result in the overlap of the resonance

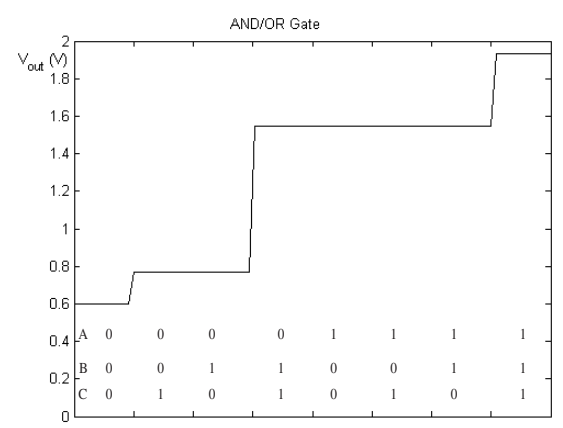

Fig. 10. A derived truth table for a three-input AND/OR gate (ie, the strong coupling limit). We conclude that this abstract model not only gives a simple yet useful expression for the I-V characteristics realized by molecular conduction, but also contains configurable parameters related to the physical geometry. As such, it is ideal for the intended purpose of nanoelectronic circuit simulation involving complex architectures such as the crossbar scheme.

\section{REFERENCES}

[1] J. Chen and M. A. Reed, "Large on-off ratios and negative differential resistance in a molecuar electronic device," Science, vol. 286, pp. 1550, 1999.

[2] C. P. Collier, E. W. Wong, M. Belohradsky, F. M. Raymo, J. F. Stoddart, P. J. Kuekes, R. S. Williams and J. R. Heath, "Electronically configurable molecular-based logic gates," Science, vol. 285, pp. 391, 1999.

[3] W. Liang, M. P. Shores, M. Bockrath, J. R. Lang and H. Park, "Kondo resonance in a single-molecule transistor," Nature, vol. 417, pp. 725, 2002.

[4] R. M. Metzger, B. Chen, U. Hopfner, M. V. Lakshmikantham, D. Vuillaume, T. Kawai, X. Wu, H. Tachibana, T. V. Hughes, H. Sakurai, J. W. Baldwin, C. Hosch, M. P. Cava, L. Brehmer and G. J. Ashwell, "Unimolecular electrical rectification in Hexadecylquinolinium tricyanoquinodimethanide," J. Am. Chem. Soc, vol. 119, pp. 10455, 1997.

[5] C. M. Fischer, M. Burghard, S. Roth and K. V. Klitzing, "Microstructured gold/Langmuir-Blodgett film/gold tunneling junctions," Appl. Phys. Lett, vol.66, pp.3331, 1995.

[6] Y. Huang, X. F. Duan, Y. Cui, L. J. Lauhon, K. H. Kim and C. M. Lieber, "Logic gates and computation from assembled nanowire building blocks," Science, vol. 294, pp.1313, 2001.

[7] A. Bachtold, P. Hadley, T. Nakanishi and C. Dekker, "Logic circuit with carbon nanotube transistor," Science, vol. 294, pp. 1317, 2001.

[8] C. Collier, G. Mattersteig, E. Wong, Y. Luo, K. Beverly, J. Sampaio, F. Raymo, J. Stoddart and J. Heath, "A [2]catenane-based solid state reconfigurable switch," Science, vol.289, pp. 1172, 2000.

[9] Y. Chen, G. Y. Jung, D. A. Ohlberg, X. Li, D. R. Stewart, J. O. Jeppesen, K. A. Nielsen, J. F. Stoddart and R. S. Williams, "Nanoscale molecularswitch crossbar circuits," Nanotechnology, vol. 14, pp.462, 2003.

[10] S. C. Goldstein and M. Budiu, "Nanofabrics: spatial computing using molecular nanoelectronics," in Proc. 28th int. Symp. Computer Architecture, pp.178, 2001

[11] A. R. Pease, J. O. Jeppesen, J. F. Stoddart, Y. Luo, C. P. Collier and J. R. Heath, "Switching devices based on interlocked molecules," Acc. Chem. Res., vol. 34, pp. 433, 2001.

[12] T. Rueckes, K. Kim, E. Joselevich, G. Y. Tseng, C. Cheung, and C. M. Lieber," Carbon nanotube-based nonvolatile random access memory for molecular computing," Science, vol. 289, pp.94, 2000.

[13] M. Ziegler and M. Stan, "Design and analysis of crossbar circuits for molecular nanoelectronics," presented at the IEEE Conf. Nanotechnology(IEEE-NANO), Washington, DC, 2002.

[14] S. Datta, Quantum Transport: Atom to Transistor. Cambridge University Press, 2005.

[15] R. Landauer, "Can a length of perfect conductor have a resistance?" Phys. Lett, vol. 85A, pp. 91, 1981.

[16] S. Sanvito, C. J. Lambert, J. H. Jefferson and A. M. Bratkovsky, "general Green's function formulism for transport calculations with spd Hamiltonians and giant magnetorisistance in $\mathrm{Co}-$ and $\mathrm{Ni}$ - based magnetic multilayers," Phys. Rev. B, vol. 59, pp. 936, 1999.

[17] C. Kergueris, J. P. Bourgoin and S. Palacin, "Electron transport through a metal-molecule-metal junction," Phys. Rev. B, vol. 59, pp. 505, 1999.

[18] P. E. Kornilovitch and A. M bratkovsky, "Orentational dependence of current through molecular films," Phys. Rev. B, vol. 64, pp. 195413, 2001.

[19] Z. Yan and M. J. Dear, "New RTD large-signal DC suitable for PSPICE," IEEE Trans. Computer-Aided Design, vol. 14, pp. 167, 1995.

[20] J. N. Schulman, H. J. De Los Santos, and D. H. Chow, "Physics based RTD current-voltage equation," IEEE Electron Device Lett., vol. 17, pp.220, 1996.

[21] M. Ziegler, G. Rose, and M. Stan, "A universal device model for nanoelectronic circuit simulation," presented at the IEEE Conf. Nanotechnology(IEEE-NANO), Washington, DC, 2002. 